\title{
Nanofluidic Charge Transport under Strong Electrostatic Coupling Conditions
}

\author{
Sahin Buyukdagli*
}

Cite This: J. Phys. Chem. B 2020, 124, 11299-11309

\begin{abstract}
ACCESS
Џlll Metrics \& More

Article Recommendations

Supporting Information

ABSTRACT: The comprehensive depiction of the many-body effects governing nanoconfined electrolytes is an essential step for the conception of nanofluidic devices with optimized performance. By incorporating self-consistently multivalent charges into the Poisson-Boltzmann equation dressed by a background monovalent salt, we investigate the impact of strong-coupling electrostatics on the nanofluidic transport of electrolyte mixtures. We find that the experimentally observed negative streaming currents in anionic nanochannels originate from the collective effect of $\mathrm{Cl}^{-}$attraction by the interfacially adsorbed multivalent cations and the no-slip layer reducing the hydrodynamic contribution of these cations to the net current. The like-charge current condition emerging from this collective mechanism is shown to be the reversal of the average potential within the no-slip zone. Applying the formalism to surface-coated membrane nanoslits located in the giant dielectric permittivity regime, we reveal a new type of streaming current activated by attractive polarization forces. Under the effect of these forces, multivalent ions added to the $\mathrm{KCl}$ solution set a charge separation and generate a counterion current between the neutral slit walls where the pure $\mathrm{KCl}$ conductivity vanishes. The adjustability of the current characteristics solely via the valency and amount of the added multivalent ions identifies the underlying process as a promising mechanism for nanofluidic ion separation purposes.

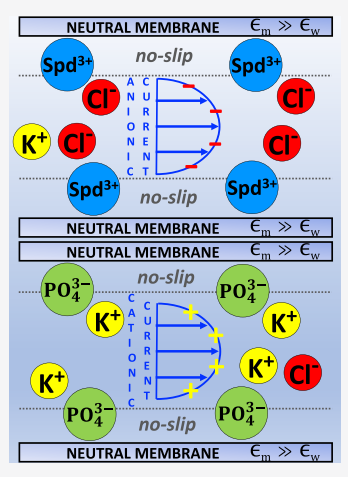

\section{INTRODUCTION}

Charge transport under nanoscale forces plays a critical role in vital biological processes and various nanoscale applications. From ion exchange between cells and their surrounding aqueous medium $^{1}$ to nanofluidic energy conversion ${ }^{2-6}$ and water desalination, ${ }^{7,8}$ diverse nanoscale mechanisms involving the transport of confined liquids are regulated by the collective effect of electrostatic, hydrodynamic, and steric forces. The accurate characterization of these nanoscale forces is thus crucial for the comprehension of living matter and the optimization of nanotechnological approaches. This need continues to motivate intensive research work dealing with the high complexity of the nanoconfined liquids associated with the diversity of the underlying interactions.

The minimal approach to tackle this complex transport problem consists of solving the coupled electrostatic PoissonBoltzmann (PB) and hydrostatic Stokes equations. In monovalent salt solutions governed by mean-field (MF) electrostatics, this hybrid formalism has been successfully verified by pressure-driven transport experiments ${ }^{9}$ and also used to develop strategies for electrokinetic energy conversion from hydrodynamic to electrical power ${ }^{10-13}$ as well as nanofluidic ion separation under hydrostatic and electrical driving forces. ${ }^{14}$ Subsequently, this approach has been upgraded by incorporation of the direct hydrodynamic effect of ions ${ }^{15}$ and polyelectrolytes ${ }^{16}$ on electroosmotic (EO) flows. Later studies showed that the explicit contribution of the mobile charges to the interfacial liquid viscosity can induce EO flow reversal even in monovalent electrolytes governed by MF electrostatics.
For ions of valency $q_{\mathrm{c}}$ interacting with a membrane of surface charge density $\sigma_{\mathrm{m}}$, the importance of charge correlations responsible for the deviation from the MF electrostatics is characterized by the dimensionless electrostatic coupling parameter $\Xi=2 \pi q_{\mathrm{C}}^{3} l_{\mathrm{B}}^{2} \sigma_{\mathrm{m}}$ where $l_{\mathrm{B}} \approx 7 \AA$ is the Bjerrum length. ${ }^{17}$ In the case of silica nanochannels of characteristic wall charge density $\sigma_{\mathrm{m}} \approx 1.0 \mathrm{e} / \mathrm{nm}^{2},{ }^{18}$ the coupling parameter exhibits a broad variation between the weak-coupling (WC) regime $\Xi \approx 3$ of monovalent salt solutions and the strong-coupling (SC) regime $\Xi \approx 195$ of quadrivalent charges such as spermine $\left(\mathrm{Spm}^{4+}\right)$ molecules. Consequently, MF electrohydrodynamic theories capable of describing monovalent charge transport fail at explaining multivalent charge-driven exotic transport behavior, such as negative streaming currents through anionic slits $^{18}$ and the electrophoretic mobility of anionic macromolecules along the applied electric field. ${ }^{19-21}$ This deficiency indicates the necessity to use correlation-corrected theories for the rectification of the MF interaction picture.

Like-charge streaming current formation by multivalent counterion addition has been previously studied within the density functional theory (DFT) capable of accounting for

$\begin{array}{ll}\text { Received: } & \text { October 25, } 2020 \\ \text { Revised: } & \text { November 11, } 2020 \\ \text { Published: } & \text { November 24, } 2020\end{array}$ 
charge correlations. ${ }^{18,22,23}$ Despite the confirmed accuracy of the DFT approach, the complexity of its numerical implementation necessitates the development of alternative beyond-MF formalisms offering analytical transparency and thereby providing direct physical insight into exotic electrostatic phenomena.

Along these lines, the field theory approach to charge liquids has been a leading alternative to DFT. The formulation of the electrostatic field theory has been mainly motivated by the observation of seemingly counterintuitive equilibrium phenomena, such as macromolecular like-charge attraction and opposite-charge repulsion driven by ion correlations. ${ }^{24-26}$ The field-theoretic investigation of charge correlations has been initiated with the solution of the correlation-corrected PB-like equations. $^{27,28}$ Then, a consistent one-loop (11) theory of counterion liquids was developed by Netz and Orland. ${ }^{29}$ This has been subsequently extended to salt solutions symmetrically distributed around a plane ${ }^{30}$ and to mixed electrolytes confined to slits and nanopores. ${ }^{21,31-33}$

The limitation of the aforementioned theories to the electrostatic WC regime stems from the underlying perturbative inclusion of the ion correlations in terms of the coupling parameter $\Xi$. Consequently, in the SC regime of tri- and quadrivalent ions where $\Xi \gg 1$, charge correlations cease to be perturbative and the loop expansion loses its validity. In order to overcome this limitation, Moreira and Netz developed an SC theory of counterion liquids derived from the expansion of the liquid grand potential in the inverse coupling parameter $\Xi^{-1} \cdot{ }^{17}$ It is noteworthy that this SC formalism can be equivalently obtained from the virial expansion of the liquid partition function in terms of the counterion fugacity.

Biological systems are characterized by the coexistence of monovalent salt and multivalent ions. The understanding of these systems thus requires electrostatic formalisms able to take into account the multiple coupling strengths governing composite electrolytes. Motivated by this need, the SC theory ${ }^{19}$ has been extended by the incorporation of a monovalent salt background at the linear-MF level. ${ }^{34,35}$ Subsequently, this dressed-ion theory has been upgraded by us via the inclusion of an additional loop correction for the monovalent salt component. ${ }^{36}$ Finally, through a Gaussian-level variational treatment of the WC salt, and the inclusion of the SC multivalent ions via a virial expansion, we derived a fully selfconsistent formalism of complex electrolytes called the SCdressed Schwinger-Dyson (SCSD) theory. ${ }^{37}$

In this article, we develop an SC-dressed PB (SCPB) formalism corresponding to a simplified version of the SCSD theory. Based on a restricted closure of the Schwinger-Dyson (SD) equation, this simplification enables us to account for the previously neglected ionic hard-core (HC) interactions. ${ }^{37}$ Within this formalism, we review the electrohydrodynamic mechanism behind the multivalent ion-driven streaming current inversion in anionic silica channels. ${ }^{9,22,23,38}$ We characterize the collective effect of the multivalent cations responsible for the membrane charge inversion (CI) and the no-slip constraint limiting the hydrodynamic mobility of these ions. We identify as well the resulting electrostatic condition for the onset of current inversion and consider the effect of repulsive polarization forces neglected in earlier works.

The continuous demand for electronic devices of reduced size necessitates the fabrication of micro capacitors with polymerbased materials of high dielectric permittivity. This technical requirement has been met by coating the surface of the polymer matrices by carbon nanotubes (CNTs), enabling the enhancement of the substrate permittivity from the dielectric insulator $\varepsilon_{\mathrm{m}} \approx 2$ to the giant permittivity regime $\varepsilon_{\mathrm{m}} \approx 10^{3.39}$. The second part of our work probes the potential of surface-coated membrane pores for nanofluidic charge transport purposes. Therein, we reveal a new current generation mechanism triggered by SC interactions. Namely, under the effect of attractive polarization forces emerging in the giant permittivity regime, multivalent cations (anions) added to a $\mathrm{KCl}$ solution result in a charge separation and generate a negative (positive) streaming current through interfacially neutral slits.

Due to the possibility to tune the sign and magnitude of this counterion current via the type and concentration of the added multivalent ions, our prediction presents itself as a useful mechanism for nanofluidic ion separation and water purification purposes. We characterize as well the impact of the same attractive polarization forces on the ionic composition of voltage-driven currents. Finally, a direct mapping from the SCPB to the dressed-ion formalism ${ }^{34,35}$ is presented in the Supporting Information.

\section{THEORY}

II.A. Field-Theoretic Coulombic Model. The charge composition of the system is depicted in Figure 1. The

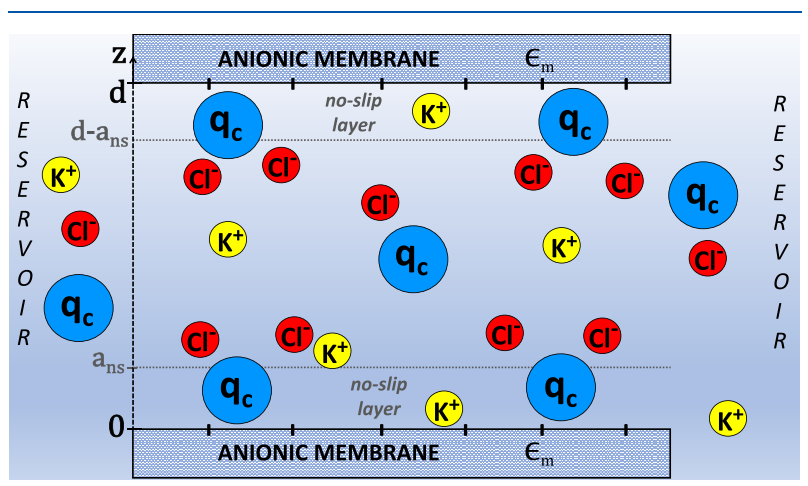

Figure 1. Schematic depiction of the slit pore with thickness $d$, length $L$, and width $w$. The slit located in an anionic membrane of permittivity $\varepsilon_{\mathrm{m}}$ confines the electrolyte $\mathrm{KCl}+\mathrm{X}^{q_{c}}$ of permittivity $\varepsilon_{\mathrm{w}}$ including the multivalent ions $X^{q_{c}}$ of valency $q_{c}$. The charge configuration in the figure assumes $q_{\mathrm{c}}>0$.

electrolyte is composed of the monovalent salt $\mathrm{KCl}$ including ions with valencies $q_{ \pm}= \pm 1$ and bulk concentrations $n_{ \pm b}$, and a multivalent ion species with bulk concentration $n_{\mathrm{cb}}$ and valency $q_{c}$ of arbitrary sign. The charge configuration in Figure 1 corresponds to the specific case of multivalent cations $\left(q_{\mathrm{c}}>0\right)$. The electrolyte is confined to a slit pore of thickness $d$ located in a membrane of dielectric permittivity $\varepsilon_{\mathrm{m}}$. The pore is composed of two anionic planes of surface charge density $-\sigma_{\mathrm{m}}$, lateral length $L$, and width $w$. Neglecting the finite size effects associated with the lateral wall boundaries where the slit is connected to an ion reservoir, the surface charge distribution is given by

$$
\sigma(\mathbf{r})=-\sigma_{\mathrm{m}}[\delta(z)+\delta(d-z)]
$$

The ions interact via the repulsive $\mathrm{HC}$ potential $w\left(\mathbf{r}-\mathbf{r}^{\prime}\right)$ and the electrostatic Coulombic potential $v_{\mathrm{c}}\left(\mathbf{r}, \mathbf{r}^{\prime}\right)$. The HC potential is defined as $w\left(\mathbf{r}-\mathbf{r}^{\prime}\right)=\infty$ if $\left\|\mathbf{r}-\mathbf{r}^{\prime}\right\| \leq 2 a_{\mathrm{hc}}$ and $w(\mathbf{r}$ $\left.-\mathbf{r}^{\prime}\right)=0$ for $\left\|\mathbf{r}-\mathbf{r}^{\prime}\right\|>2 a_{\mathrm{hc}}$ with the same HC radius $a_{\mathrm{hc}}$ assumed 
for all ionic species. The Coulombic potential is defined in terms of its inverse

$$
v_{\mathrm{c}}^{-1}\left(\mathbf{r}, \mathbf{r}^{\prime}\right)=-\frac{k_{\mathrm{B}} T}{\mathrm{e}^{2}} \nabla \varepsilon(\mathbf{r}) \nabla \delta\left(\mathbf{r}-\mathbf{r}^{\prime}\right)
$$

where $k_{\mathrm{B}}$ is the Boltzmann constant and e is the electron charge; the liquid temperature is $T=300 \mathrm{~K}$, and the dielectric permittivity profile reads

$$
\varepsilon(\mathbf{r})=\varepsilon_{\mathrm{m}}[\theta(-z)+\theta(z-d)]+\varepsilon_{\mathrm{w}} \theta(z) \theta(d-z)
$$

with the dielectric permittivity of the solvent $\varepsilon_{\mathrm{w}}=80$.

By introducing two Hubbard-Stratonovich transformations associated with the fluctuating potentials $\phi(\mathbf{r})$ and $\psi(\mathbf{r})$ for the pairwise Coulombic and $\mathrm{HC}$ ion interactions, respectively, the grand-canonical partition function of the system can be recast as the functional integral ${ }^{40}$

$$
\mathrm{Z}_{\mathrm{G}}=\int \mathcal{D} \phi \mathcal{D} \psi e^{-\beta H[\phi, \psi]}
$$

In eq 4 , the Hamiltonian functional reads $H[\phi, \psi]=H_{\mathrm{s}}[\phi, \psi]$ $+H_{c}[\phi, \psi]$ where the solvent-implicit monovalent salt and multivalent ion components are defined as

$$
\begin{aligned}
\beta H_{\mathrm{s}}[\phi, \psi]= & \frac{k_{\mathrm{B}} T}{2 \mathrm{e}^{2}} \int \mathrm{d} \mathbf{r} \varepsilon(\mathbf{r})[\nabla \phi(\mathbf{r})]^{2} \\
& -i \int \mathrm{d} \mathbf{r} \sigma(\mathbf{r}) \phi(\mathbf{r}) \\
& +\frac{1}{2} \int \mathrm{d} \mathbf{r} \mathrm{d} \mathbf{r}^{\prime} \psi(\mathbf{r}) w^{-1}\left(\mathbf{r}-\mathbf{r}^{\prime}\right) \psi\left(\mathbf{r}^{\prime}\right) \\
& -\sum_{i= \pm} \int \mathrm{d} \mathbf{r} \hat{\rho}_{i}(\mathbf{r}) \\
\beta H_{\mathrm{c}}[\phi, \psi]= & -\int \mathrm{d} \mathbf{r} \hat{\rho}_{\mathrm{c}}(\mathbf{r})
\end{aligned}
$$

The first three terms on the r.h.s. of eq 5 correspond to the solvent free energy, the contribution from the fixed membrane charge $\sigma(\mathbf{r})$, and the Gaussian field contribution from the pairwise HC interactions. Then, the ion density term $\hat{\rho}_{i}(\mathbf{r})$ in eqs 5-6 is the contribution from the mobile salt charges $(i= \pm)$ and multivalent ions $(i=\mathrm{c})$. Therein, the fluctuating ion density of the species $i$ with fugacity $\Lambda_{i}$ reads

$$
\hat{\rho}_{i}(\mathbf{r})=\Lambda_{i} e^{-V_{i}(\mathbf{r})+i q_{i} \phi(\mathbf{r})+i \psi(\mathbf{r})}
$$

where $V_{i}(\mathbf{r})$ stands for the steric potential restricting the phase space accessible to the ions and enabling the derivation of their average density from the grand potential $\beta \Omega_{\mathrm{G}}=-\ln Z_{\mathrm{G}}$ via the thermodynamic identity

$$
n_{i}(\mathbf{r})=\frac{\delta\left[\beta \Omega_{\mathrm{G}}\right]}{\delta V_{i}(\mathbf{r})}=\left\langle\hat{\rho}_{i}(\mathbf{r})\right\rangle
$$

In eq 8 , the bracket notation designates the statistical average of the functional $F[\phi, \psi]$ over the fluctuations of the potentials $\phi(\mathbf{r})$ and $\psi(\mathbf{r})$, i.e.

$$
\langle F[\phi, \psi]\rangle=\frac{1}{Z_{\mathrm{G}}} \int \mathcal{D} \phi \mathcal{D} \psi e^{-\beta H[\phi, \psi]} F[\phi, \psi]
$$

The steric constraint $a_{\mathrm{st}}<z<d-a_{\mathrm{st}}$ imposing on the ions the closest approach distance $a_{\mathrm{st}}$ to the slit walls will be taken as $e^{-V_{i}(\mathbf{r})}=\theta\left(z-a_{\mathrm{st}}\right) \theta\left(d-a_{\mathrm{st}}-z\right)$.

II.B. Derivation of the Electrostatic SCPB Equation. Based on the SD identity, a simplified version of the SCSD approach $^{37}$ will be derived. For the derivation of the SD equation, one expresses first the variation of the partition function (eq 4) by an infinitesimally small potential shift $\delta \phi(\mathbf{r})$ as $\delta Z_{\mathrm{G}}=\int \mathcal{D} \phi \mathcal{D} \psi e^{-\beta H[\phi+\delta \phi, \psi]}-Z_{\mathrm{G}}$. In the remainder of the article, the notation will be simplified by omitting the potential dependence of the functionals. Expanding now the r.h.s. of the functional integral above at the linear order in $\delta \phi(\mathbf{r})$, one obtains $\delta Z_{\mathrm{G}}=-\beta \mathrm{Z}_{\mathrm{G}} \int \mathrm{d} \mathbf{r} \delta \phi(\mathbf{r})\langle\delta H / \delta \phi(\mathbf{r})\rangle$. Then, one notes that the shift $\delta \phi(\mathbf{r})$ in the same functional integral can be absorbed into the redefinition of the functional measure $\mathcal{D} \phi$. This implies the invariance of the partition function $Z_{\mathrm{G}}$ under the potential shift, i.e., $\delta Z_{\mathrm{G}}=0$. This finally yields the formally exact $\mathrm{SD}$ identity corresponding to the equation of state for the average potential

$$
\left\langle\frac{\delta H}{\delta \phi(\mathbf{r})}\right\rangle=0
$$

At this point, we introduce the SC treatment of the dilute multivalent ions equivalent to a low fugacity expansion of the grand potential. ${ }^{17}$ First, by Taylor-expanding the SD eq 10 together with eq 9 at the linear order in the counterion density $\hat{\rho}_{c}(\mathbf{r})$, one obtains

$$
\begin{aligned}
\left\langle\frac{\delta H}{\delta \phi(\mathbf{r})}\right\rangle= & \left\langle\frac{\delta H}{\delta \phi(\mathbf{r})}\right\rangle_{\mathrm{s}}-\left\{\left\langle\frac{\delta H_{\mathrm{s}}}{\delta \phi(\mathbf{r})} H_{\mathrm{c}}\right\rangle_{\mathrm{s}}\right. \\
& \left.-\left\langle\frac{\delta H_{\mathrm{s}}}{\delta \phi(\mathbf{r})}\right\rangle_{\mathrm{s}}\left\langle H_{\mathrm{c}}\right\rangle_{\mathrm{s}}\right\} \\
= & 0
\end{aligned}
$$

In eq 11 , the average of the general functional $F[\phi, \psi]$ with the salt Hamiltonian (eq 5) has been defined as

$$
\langle F\rangle_{\mathrm{s}}=\frac{1}{Z_{\mathrm{s}}} \int \mathcal{D} \phi \mathcal{D} \psi e^{-\beta H_{\mathrm{s}}} F
$$

where the salt partition function reads $Z_{\mathrm{s}}=\int \mathcal{D} \phi \mathcal{D} \psi e^{-\beta H_{s}}$. Moreover, at the same order $O\left(\hat{\rho}_{\mathrm{c}}\right)$, the SC expansion of the ion density functions (eq 8 ) gives

$$
\begin{aligned}
& n_{ \pm}(\mathbf{r})=\left\langle\hat{\rho}_{ \pm}(\mathbf{r})\right\rangle_{\mathrm{s}}+\int \mathrm{d} \mathbf{r}_{\mathrm{c}}\left[\left\langle\hat{\rho}_{ \pm}(\mathbf{r}) \hat{\rho}_{\mathrm{c}}\left(\mathbf{r}_{\mathrm{c}}\right)\right\rangle_{\mathrm{s}}-\left\langle\hat{\rho}_{ \pm}(\mathbf{r})\right\rangle_{\mathrm{s}}\left\langle\hat{\rho}_{\mathrm{c}}\left(\mathbf{r}_{\mathrm{c}}\right)\right\rangle_{\mathrm{s}}\right] \\
& n_{\mathrm{c}}(\mathbf{r})=\left\langle\hat{\rho}_{\mathrm{c}}(\mathbf{r})\right\rangle_{\mathrm{s}}
\end{aligned}
$$

Inserting now the Hamiltonian components (eqs 5 and 6) into eq 11, the virial-expanded SD equation follows as

$$
\begin{aligned}
& \frac{k_{\mathrm{B}} T}{\mathrm{e}^{2}}\langle\nabla \varepsilon(\mathbf{r}) \nabla \phi(\mathbf{r})\rangle_{\mathrm{s}}+i \sigma(\mathbf{r})+i \sum_{i= \pm, \mathrm{c}} q_{i} n_{i}(\mathbf{r}) \\
&=-\frac{k_{\mathrm{B}} T}{\mathrm{e}^{2}} \int \mathrm{d}_{\mathrm{c}}\left\langle\left\langle\hat{\rho}_{\mathrm{c}}\left(\mathbf{r}_{\mathrm{c}}\right) \nabla \varepsilon(\mathbf{r}) \nabla \phi(\mathbf{r})\right\rangle_{\mathrm{s}}\right. \\
&\left.-\left\langle\hat{\rho}_{\mathrm{c}}\left(\mathbf{r}_{\mathrm{c}}\right)\right\rangle_{\mathrm{s}}\langle\nabla \varepsilon(\mathbf{r}) \nabla \phi(\mathbf{r})\rangle_{\mathrm{s}}\right]
\end{aligned}
$$


In order to evaluate the field averages in eq 15 associated with the WC fluctuations of the monovalent salt around the MF PB solution, the salt Hamiltonian (eq 5) will be approximated by the following Gaussian functional

$$
\begin{aligned}
H_{\mathrm{s}} \approx & \frac{1}{2} \int_{\mathbf{r}, \mathbf{r}^{\prime}}\left[\phi-i \phi_{s}\right]_{\mathbf{r}} G^{-1}\left(\mathbf{r}, \mathbf{r}^{\prime}\right)\left[\phi-i \phi_{\mathbf{s}}\right]_{\mathbf{r}^{\prime}} \\
& +\frac{1}{2} \int_{\mathbf{r}, \mathbf{r}^{\prime}} \psi(\mathbf{r}) w^{-1}\left(\mathbf{r}-\mathbf{r}^{\prime}\right) \psi\left(\mathbf{r}^{\prime}\right)
\end{aligned}
$$

Equation 16 includes the inverse of the monovalent saltdressed Debye-Hückel (DH) Green's function

$$
G^{-1}\left(\mathbf{r}, \mathbf{r}^{\prime}\right)=v_{\mathrm{c}}^{-1}\left(\mathbf{r}, \mathbf{r}^{\prime}\right)+\sum_{i= \pm} q_{i}^{2} n_{i \mathrm{~b}} e^{-V_{i}(\mathbf{r})} \delta\left(\mathbf{r}-\mathbf{r}^{\prime}\right)
$$

and the salt-screened average potential $\phi_{\mathrm{s}}(\mathbf{r})$, solving the SD eq 15. Using now the identity

$$
\int \mathrm{d} \mathbf{r}^{\prime \prime} G^{-1}\left(\mathbf{r}, \mathbf{r}^{\prime \prime}\right) G\left(\mathbf{r}^{\prime \prime}, \mathbf{r}^{\prime}\right)=\delta\left(\mathbf{r}-\mathbf{r}^{\prime}\right)
$$

with eqs 2 and 17, the differential equation solved by the $\mathrm{DH}$ Green's function follows as

$$
\left[\nabla \varepsilon(\mathbf{r}) \nabla-\varepsilon(\mathbf{r}) \kappa^{2}(r)\right] G\left(\mathbf{r}, \mathbf{r}^{\prime}\right)=-\frac{\mathrm{e}^{2}}{k_{\mathrm{B}} T} \delta\left(\mathbf{r}-\mathbf{r}^{\prime}\right)
$$

In eq 19 , we introduced the $\mathrm{DH}$ screening parameter

$$
\kappa(r)=\kappa e^{-V_{i}(\mathbf{r})} ; \quad \kappa^{2}=4 \pi l_{\mathrm{B}} \sum_{i= \pm} q_{i}^{2} n_{i \mathrm{~b}}
$$

The solution of the kernel (eq 19) in the planar geometry of the nanoslit is reported in the Supporting Information.

Computing the field averages in eqs $13-15$ with the Hamiltonian (eq 16), the SCPB equation (eq 15) becomes

$$
\begin{aligned}
& \frac{k_{\mathrm{B}} T}{\mathrm{e}^{2}} \nabla_{\mathbf{r}} \cdot \varepsilon(\mathbf{r}) \nabla_{\mathbf{r}}\left\{\phi_{\mathrm{s}}(\mathbf{r})+q_{\mathrm{c}} \int \mathrm{d} \mathbf{r}_{\mathrm{c}} n_{\mathrm{c}}\left(\mathbf{r}_{\mathrm{c}}\right) G\left(\mathbf{r}_{\mathrm{c}}, \mathbf{r}\right)\right\} \\
& \quad+\sum_{i= \pm, \mathrm{c}} q_{i} n_{i}(\mathbf{r})+\sigma(\mathbf{r}) \\
& \quad=0
\end{aligned}
$$

with the ion densities

$$
\begin{aligned}
n_{ \pm}(\mathbf{r})= & \Lambda_{ \pm} e^{-w(0) / 2-V_{ \pm}(\mathbf{r})-q_{ \pm} \phi_{\mathrm{s}}(\mathbf{r})-q_{ \pm}^{2} G(\mathbf{r}, \mathbf{r}) / 2} \\
& \times\left\{1+\int \mathrm{d} \mathbf{r}_{\mathrm{c}} n_{\mathrm{c}}\left(\mathbf{r}_{\mathrm{c}}\right) f_{ \pm}\left(\mathbf{r}, \mathbf{r}_{\mathrm{c}}\right)\right\} \\
n_{\mathrm{c}}(\mathbf{r})= & \Lambda_{\mathrm{c}} e^{-w(0) / 2-V_{\mathrm{c}}(\mathbf{r})-q_{\mathrm{c}} \phi_{\mathrm{s}}(\mathbf{r})-q_{\mathrm{c}}^{2} G(\mathbf{r}, \mathbf{r}) / 2}
\end{aligned}
$$

and the Mayer function

$$
f_{i}\left(\mathbf{r}, \mathbf{r}_{\mathrm{c}}\right)=e^{-q_{i} q_{\mathrm{c}} G\left(\mathbf{r}, \mathbf{r}_{\mathrm{c}}\right)-w\left(\mathbf{r}-\mathbf{r}_{\mathrm{c}}\right)}-1
$$

Evaluating eqs 22 and 23 in the bulk reservoir where $\sigma(\mathbf{r})=0$, $\phi_{\mathrm{s}}(\mathbf{r})=0$, and $n_{i}(\mathbf{r})=n_{\mathrm{b}}$ for $i=\{ \pm, \mathrm{c}\}$ and $G\left(\mathbf{r}, \mathbf{r}^{\prime}\right)=G_{\mathrm{b}}\left(\mathbf{r}-\mathbf{r}^{\prime}\right)$ with the bulk Green's function $G_{\mathrm{b}}(r)=4 \pi l_{\mathrm{B}} e^{-\kappa r} / r$ and plugging the resulting ion fugacities back into eqs 22 and 23, the ion densities become at the order $O\left(n_{\mathrm{cb}}\right)$

$$
\begin{aligned}
& n_{ \pm}(\mathbf{r})=n_{ \pm \mathrm{b}} k_{ \pm}(\mathbf{r})\left[1+n_{\mathrm{cb}} T_{ \pm}(\mathbf{r})\right], \\
& n_{\mathrm{c}}(\mathbf{r})=n_{\mathrm{cb}} k_{\mathrm{c}}(\mathbf{r}) .
\end{aligned}
$$

In eqs 25 and 26, the function

$$
k_{i}(\mathbf{r})=e^{-V_{i}(\mathbf{r})-q_{i} \phi_{s}(\mathbf{r})-q_{i}^{2} \delta G(\mathbf{r}) / 2}
$$

corresponds to the bare ion density including the self-energy $\delta G(\mathbf{r})=\left[G\left(\mathbf{r}, \mathbf{r}^{\prime}\right)-G_{\mathrm{b}}\left(\mathbf{r}-\mathbf{r}^{\prime}\right)\right]_{\mathbf{r}^{\prime} \rightarrow \mathbf{r}}$. Moreover, eq 25 involves the many-body potentials incorporating the direct interactions of the multivalent counterions with the salt ions

$$
T_{i}(\mathbf{r})=\int \mathrm{d} \mathbf{r}_{\mathrm{c}}\left[k_{\mathrm{c}}\left(\mathbf{r}_{\mathrm{c}}\right) f_{i}\left(\mathbf{r}, \mathbf{r}_{\mathrm{c}}\right)-f_{i \mathrm{~b}}\left(\mathbf{r}-\mathbf{r}_{\mathrm{c}}\right)\right]
$$

with the bulk limit of the Mayer function (eq 24)

$$
f_{i \mathrm{~b}}\left(\mathbf{r}-\mathbf{r}_{\mathrm{c}}\right)=e^{-q_{i} q_{\mathrm{c}} G_{\mathrm{b}}\left(\mathbf{r}-\mathbf{r}_{\mathrm{c}}\right)-w\left(\mathbf{r}-\mathbf{r}_{\mathrm{c}}\right)}-1
$$

One notes that the function (eq 28) has the form of a nonuniform second virial coefficient $B_{2}$ weighted by the counterion density and renormalized by its bulk value. ${ }^{41}$

Finally, combining eqs 19 and 21, the SCPB equation takes the following integro-differential form

$$
\frac{k_{\mathrm{B}} T}{e^{2}} \nabla_{\mathbf{r}} \cdot \varepsilon(\mathbf{r}) \nabla_{\mathbf{r}} \phi_{\mathrm{s}}(\mathbf{r})+\sum_{i= \pm} q_{i} n_{i}(\mathbf{r})+\sigma(\mathbf{r})=-\frac{\kappa_{\mathrm{s}}^{2}(\mathbf{r})}{4 \pi l_{\mathrm{B}}} \phi_{\mathrm{c}}(\mathbf{r})
$$

where we introduced the potential component induced by all multivalent counterions in the liquid

$$
\phi_{\mathrm{c}}(\mathbf{r})=q_{\mathrm{c}} n_{\mathrm{cb}} \int \mathrm{d}^{3} \mathbf{r}_{\mathrm{c}} k_{\mathrm{c}}\left(\mathbf{r}_{\mathrm{c}}\right) G\left(\mathbf{r}, \mathbf{r}_{\mathrm{c}}\right)
$$

One notes that, in the bulk limit where $n_{i}(\mathbf{r})=n_{i \mathrm{~b}}$ and $\int_{\mathbf{r}_{\mathrm{c}}} G_{\mathrm{b}}\left(\mathbf{r}-\mathbf{r}_{\mathrm{c}}\right)=q_{+}^{2} n_{+\mathrm{b}}+q_{-2}^{2} n_{-\mathrm{b}}$, the bulk electroneutrality condition consistently follows eq 30 as

$$
q_{+} n_{+\mathrm{b}}+q_{-} n_{-\mathrm{b}}+q_{\mathrm{c}} n_{c \mathrm{~b}}=0
$$

In order to determine the net average potential, in eq 9 , we set $F=\phi(\mathbf{r})$ and linearize the result in the counterion density. One gets, at the order $O\left(\hat{\rho}_{\mathrm{c}}\right)$

$$
\langle\phi(\mathbf{r})\rangle=\langle\phi(\mathbf{r})\rangle_{\mathrm{s}}-\left[\left\langle\phi(\mathbf{r}) H_{\mathrm{c}}\right\rangle_{\mathrm{s}}-\langle\phi(\mathbf{r})\rangle_{\mathrm{s}}\left\langle H_{\mathrm{c}}\right\rangle_{\mathrm{s}}\right]
$$

Evaluating the salt averages in eq 33 , the real average potential $\Phi(\mathbf{r})=-i\langle\phi(\mathbf{r})\rangle$ follows as

$$
\Phi(\mathbf{r})=\phi_{\mathrm{s}}(\mathbf{r})+\phi_{\mathrm{c}}(\mathbf{r})
$$

Equation 34 indicates that the net average potential is given by the superposition of the salt-dressed potential $\phi_{\mathrm{s}}(\mathbf{r})$ created by the membrane charge, satisfying the SCPB equation (eq 30), and the potential component (31eq 31) induced by the multivalent counterions. ${ }^{42}$ In this work, the SCPB equation (eq 30) will be solved via a perturbative expansion in terms of the counterion concentration $n_{\mathrm{cb}}$. The details of this solution scheme are reported in the Supporting Information.

Combining eqs 21 and 34, the SCPB equation (eq. 30) can be expressed in terms of the net potential $\Phi(\mathbf{r})$ as

$$
\frac{k_{\mathrm{B}} T}{\mathrm{e}^{2}} \nabla_{\mathbf{r}} \cdot \varepsilon(\mathbf{r}) \nabla_{\mathbf{r}} \Phi(\mathbf{r})+\rho_{\mathrm{ch}}(\mathbf{r})+\sigma(\mathbf{r})=0
$$

In eq 35 , the total mobile charge density reads

$$
\rho_{\mathrm{ch}}(\mathbf{r})=\sum_{i= \pm, \mathrm{c}} q_{i} n_{i}(\mathbf{r})
$$

with the bare partition function (eq 27) taking the form $k_{i}(\mathbf{r})=$ $e^{-V_{i}(\mathbf{r})-q_{i}\left[\Phi(\mathbf{r})-\phi_{\mathrm{c}}(\mathbf{r})\right]-\mathrm{q}_{i}^{2} \delta G(\mathbf{r}) / 2}$. The alternative form (eq 35) of the SCPB equation (eq. 30) is used below for the computation of the beyond-MF ionic current. 
II.C. Computation of the lonic Current. The net current driven by an external voltage $\Delta V$ and pressure gradient $\Delta P$ through the nanoslit reads

$$
I=w \mathrm{e} \int_{a_{\mathrm{ns}}}^{d-a_{\mathrm{ns}}} \mathrm{d} z \rho_{\mathrm{ch}}(z) u_{i}(z)
$$

where the hydrodynamic no-slip radius $a_{\mathrm{ns}}$ corresponds to the characteristic thickness of the stagnant liquid layers composed of the multivalent ions strongly bound to the slit walls (see Figure $1)$. Moreover, the fluid velocity $u_{i}(z)=u_{\mathrm{T}, i}+u_{\mathrm{c}}(z)$ is given by the superposition of the conductive velocity $u_{\mathrm{T}, i}=\mu_{i}$ sign $\left(\mathrm{q}_{i}\right) \Delta V / L$ characterized by the ionic mobility $\mu_{i}{ }^{43}$ the convective velocity $u_{\mathrm{c}}(z)$ satisfying the Stokes equation $\eta u_{\mathrm{c}}$ " $(z)+\Delta P / L+E \rho_{\mathrm{ch}}(z)=0^{45}$ with the liquid viscosity $\eta=8.91 \times$ $10^{-4} \mathrm{~Pa} \mathrm{~s}$, and the external electric field $E=\Delta V / L$. Solving the Stokes equation together with the relation $\rho_{\mathrm{ch}}(z)=-\Phi^{\prime \prime}(z) /$ $4 \pi l_{\mathrm{B}}$ following the SCPB equation (eq. 35 ) and imposing the noslip conditions $u_{\mathrm{c}}\left(a_{\mathrm{ns}}\right)=0$ and $u_{\mathrm{c}}\left(d-a_{\mathrm{ns}}\right)=0$, the convective velocity follows as

$$
\begin{aligned}
u_{\mathrm{c}}(z)= & \frac{\Delta P}{2 \eta L}\left[(d-z) z-\left(d-a_{\mathrm{ns}}\right) a_{\mathrm{ns}}\right] \\
& +\mu_{\mathrm{ep}} E\left[\Phi(z)-\Phi\left(a_{\mathrm{ns}}\right)\right]
\end{aligned}
$$

with the electrophoretic transport coefficient $\mu_{\text {ep }}=\mathrm{e} /\left(4 \pi l_{\mathrm{B}} \eta\right)$. Finally, substituting eq 38 into eq 37 , using the equality $\rho_{\mathrm{ch}}(z)=$ $-\Phi^{\prime \prime}(z) / 4 \pi l_{\mathrm{B}}$, and performing integrations by parts, the net current becomes ${ }^{46}$

$$
I=K_{\mathrm{p}} \Delta P+K_{\mathrm{v}} \Delta V
$$

where the conductance components are

$$
\begin{aligned}
& K_{\mathrm{p}}=\frac{\mathrm{e} w\left(d-2 a_{\mathrm{ns}}\right)}{4 \pi l_{\mathrm{B}} L \eta}\left\{\int_{a_{\mathrm{ns}}}^{d-a_{\mathrm{ns}}} \frac{\Phi(z) \mathrm{d} z}{d-2 a_{\mathrm{ns}}}-\Phi\left(a_{\mathrm{ns}}\right)\right\} \\
& K_{\mathrm{v}}=\frac{w \mathrm{e}}{L} \int_{a_{\mathrm{ns}}}^{d-a_{\mathrm{ns}}} \mathrm{d} z\left\{\frac{\mathrm{e}}{\eta}\left[\frac{\Phi^{\prime}(z)}{4 \pi l_{\mathrm{B}}}\right]^{2}+\sum_{i= \pm, \mathrm{c}} \mu_{i}\left|q_{i}\right| n_{i}(z)\right\}
\end{aligned}
$$

Equation 40 is the streaming conductance. Then, eq 41 corresponds to the voltage-driven conductance. The first and second terms in brackets of eq 41 are the convective and conductive flow components, respectively.

\section{RESULTS AND DISCUSSION}

In this part, we apply the SCPB formalism to characterize the effect of strongly coupled multivalent charges on ion transport through nanochannels made of a silicon $(\mathrm{Si})$-based insulator $\left(\varepsilon_{\mathrm{m}}\right.$ $\left.\ll \varepsilon_{\mathrm{w}}\right)$ and surface-coated dielectric membranes $\left(\varepsilon_{\mathrm{m}} \gg \varepsilon_{\mathrm{w}}\right)$. We focus on the experimentally relevant case of slits with nanoscale thickness where the conductive component of eq 41 largely dominates the convective part. ${ }^{23,47}$ The slit width and length are set to the values of $w=50 \mu \mathrm{m}$ and $L=4.5 \mathrm{~mm}$ of the transport experiments carried out by van der Heyden et al. ${ }^{18}$

III.A. Streaming Current Reversal in Charged Slit Pores. We analyze here the experimentally observed multivalent ion-driven streaming current reversal. ${ }^{18}$ Thus, the voltage is turned-off $(\Delta V=0)$, and the membrane charge density and permittivity are set to the characteristic values $\sigma_{\mathrm{m}}=1.0 \mathrm{e} / \mathrm{nm}^{2}$ and $\varepsilon_{\mathrm{m}}=2$ of the silica nanochannels used in the transport experiments. Figure 2 shows that, in pure $\mathrm{KCl}$ solutions (solid black curve), the salt decrement enhances the $\mathrm{K}^{+}$adsorption and

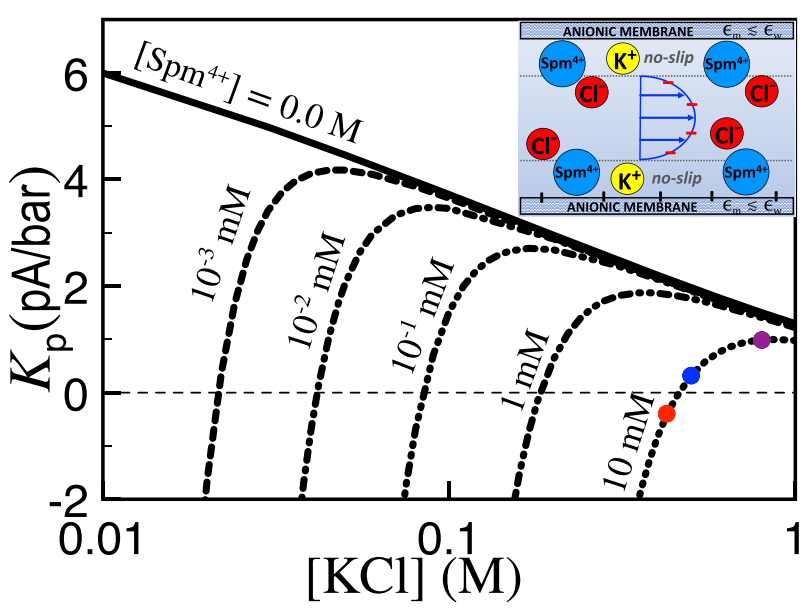

Figure 2. Streaming conductance (eq 40) vs the bulk $\mathrm{KCl}$ concentration at various $\mathrm{Spm}^{4+}$ concentration values $\left(q_{\mathrm{c}}=4\right)$. The slit charge is $\sigma_{\mathrm{m}}=1.0 \mathrm{e} / \mathrm{nm}^{2}$, ${ }^{18}$ the membrane permittivity $\varepsilon_{\mathrm{m}}=2$, the pore radius $d=50 \mathrm{~nm}$, and the steric size $a_{\mathrm{st}}=0$. The no-slip and HC radii are $a_{\mathrm{ns}}=a_{\mathrm{hc}}=3 \AA$. The picture in the inset is a qualitative depiction of the current reversal mechanism.

raises steadily the streaming conductance, i.e., $n_{+\mathrm{b}} \downarrow K_{\mathrm{P}} \uparrow$. However, if the $\mathrm{KCl}$ concentration is reduced in the presence of $\mathrm{Spm}^{4+}$ molecules, $K_{\mathrm{P}}$ initially rises monotonically by following the pure salt curve, reaching a maximum where the effect of $\mathrm{Spm}^{4+}$ ions manifests itself and starts dropping to switch from positive to negative at a characteristic $\mathrm{KCl}$ concentration $n_{\mathrm{+b}}^{*}$. Moreover, the salt concentration at the current reversal rises with the amount of $\mathrm{Spm}^{4+}$, i.e., $n_{\mathrm{cb}} \uparrow n_{+\mathrm{b}}^{*} \uparrow$. These features agree qualitatively with the transport experiments ${ }^{18}$ and earlier DFT studies $^{22,23}$ conducted with trivalent cations.

The emergence of a negative current between the anionic slit walls is typically the signature of the membrane CI. In order to probe the actual correlation between these effects, we focus first on the charge configuration at the current reversal. Figure $3 a-c$ displays the $\mathrm{Spm}^{4+}$ and monovalent ion densities (eqs 25 and 26) and the virial function (eq 28) at the salt concentrations of the dots with the same color in Figure 2. As the bulk $\mathrm{KCl}$ concentration is reduced from the positive current $K_{\mathrm{P}}>0$ (purple) to the negative current $K_{\mathrm{P}}<0$ regime (red), the suppression of the membrane charge screening drives further $\mathrm{Spm}^{4+}$ ions into the slit, i.e., $n_{+\mathrm{b}} \downarrow n_{\mathrm{c}}(z) \uparrow$. This strengthens the $\mathrm{Spm}^{4+}-\mathrm{K}^{+}$repulsion $\left(T_{+}(z) \downarrow\right)$ and the $\mathrm{Spm}^{4+}-\mathrm{Cl}^{-}$attraction $\left(T_{-}(z) \uparrow\right)$, depleting the interfacial $\mathrm{K}^{+}$ions $\left(n_{+}(z) \downarrow\right)$ and amplifying the $\mathrm{Cl}^{-}$density $\left(n_{-}(z) \uparrow\right)$ above its bulk value.

We investigate now the mechanism enabling these excess $\mathrm{Cl}^{-}$ ions to induce a negative current despite the abundance of the strongly cationic $\mathrm{Spm}^{4+}$ molecules. Figure $3 \mathrm{~d}$ displays the cumulative charge density

$$
\rho_{\mathrm{cm}}(z) \equiv \int_{0}^{z} \mathrm{~d} z^{\prime} \rho_{\mathrm{ch}}\left(z^{\prime}\right)-\sigma_{\mathrm{m}}=-\frac{\Phi^{\prime}(z)}{4 \pi l_{\mathrm{B}}}
$$

where the second equality follows the SCPB (eq 35. One sees that the apparent like-charge $\mathrm{Cl}^{-}$attraction and opposite-charge $\mathrm{K}^{+}$exclusion by the anionic membrane manifests itself as the membrane $\mathrm{CI}$. However, at the intermediate $\mathrm{KCl}$ concentration 

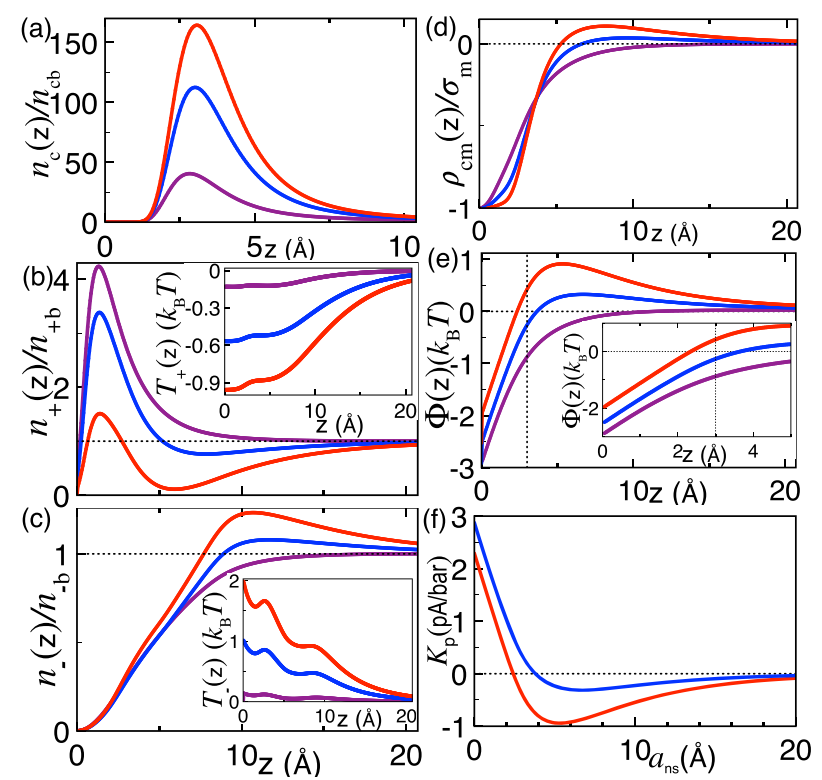

Figure 3. (a) Multivalent counterion density (eq 26). (b, c) Monovalent ion densities (eq 25) (main plots) and the salt-multivalent interaction potentials (eq 28) (insets). (d) Cumulative charge density (eq 42). (e) Net average potential (eq 34). (f) Dependence of the conductance on the no-slip radius $a_{\mathrm{ns}}$. The salt concentration values of the curves corresponding to the dots of the same color in Figure 2 are $n_{+\mathrm{b}}=0.8 \mathrm{M}$ (purple), 0.5 M (blue), and 0.425 M (red).

(blue) where CI is noticeable $\left(\rho_{\mathrm{cm}}(z)>0\right)$, the conductance is not yet inverted $\left(K_{\mathrm{P}}>0\right)$. This indicates the absence of one-toone mapping between $\mathrm{CI}$ and current reversal. ${ }^{48}$

For an analytical insight into the actual causality between CI and current reversal, one should note that, in nanofluidic experiments where one typically has $\kappa d \gg 1$, the integral part of the conductance (eq 40) is negligible, i.e.

$$
K_{\mathrm{p}} \approx-\frac{\mathrm{e} w\left(d-2 a_{\mathrm{ns}}\right)}{4 \pi l_{\mathrm{B}} L \eta} \Phi\left(a_{\mathrm{ns}}\right)
$$

Equation 43 implies that current inversion requires the reversal of the zeta potential $\Phi\left(a_{\mathrm{ns}}\right)$ at the no-slip distance $z=a_{\mathrm{ns}}$ rather than the cumulative charge (eq 42) or field $\Phi^{\prime}(z)$ at an arbitrary distance. Figure $3 e$ indeed shows that the transition from positive (blue) to negative current (red) is accompanied with the shift of the potential reversal point into the no-slip region $z<3 \AA$.

The requirement of potential inversion within the stagnant fluid layer indicates that current reversal is favored by the broadness of the no-slip region. Figure $3 \mathrm{f}$ confirms this point as the no-slip distance $a_{\mathrm{ns}}$ increases from zero, the positive conductance drops and turns to negative before decaying due to the increasing overlap of the no-slip region with the $\mathrm{Cl}^{-}$layer. In Figure 4, the electrohydrodynamic mechanism behind this peculiarity is illustrated in terms of the charge density (eq 36) and flux and the integral of the flux corresponding to the cumulative current

$$
j_{\mathrm{cm}}(z)=w \mathrm{e} \int_{a_{\mathrm{ns}}}^{z} \mathrm{~d} z \rho_{\mathrm{ch}}\left(z^{\prime}\right) u_{\mathrm{c}}\left(z^{\prime}\right)
$$

First, Figure $4 \mathrm{a}$ shows that the $\mathrm{Spm}^{4+}$ ions located in the stagnant fluid region $z \leq a_{\mathrm{ns}}$ and responsible for the strongly positive electrostatic charge density $\rho_{\mathrm{ch}}(z)$ do not contribute to

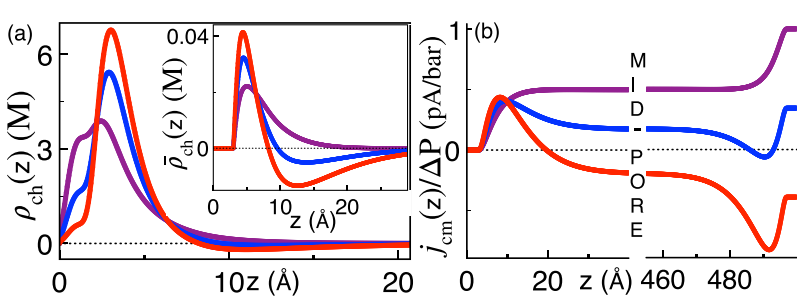

Figure 4. (a) Charge density (eq 36) (main plot) and normalized charge flux $\bar{\rho}_{\mathrm{ch}}(z)=\rho_{\mathrm{ch}}(z) u_{\mathrm{c}}(z) / u_{\mathrm{c}}(d / 2)$ (inset). (b) Cumulative current density (eq 44). Model parameters for each color are the same as in Figure 3.

the hydrodynamic charge flux $\bar{\rho}_{\mathrm{ch}}(z)$. Then, by comparing the maxima and minima in the main plot and the inset, one notes that the Poisseuille velocity (eq 38) rising steadily in the subsequent liquid region attenuates the positive charge contribution from the mobile $\mathrm{Spm}^{4+}$ ions at $z>a_{\mathrm{ns}}$, and enhances the anionic contribution of the next $\mathrm{Cl}^{-}$layer where $\rho_{\mathrm{ch}}(z)<0$.

To summarize, the $\mathrm{Spm}^{4+}$ layer around each no-slip boundary acts as an effective positive surface charge attracting mobile $\mathrm{Cl}^{-}$ anions without fully contributing itself to the charge flow. In Figure $3 \mathrm{f}$, the emergence of the anionic current by the increase of the no-slip length originates precisely from the enlargement of this cation layer with reduced mobility. Figure $4 \mathrm{~b}$ shows that, as the salt decrement enhances the adsorption of these counterions by the slit, the mobile $\mathrm{Cl}^{-}$excess set by the counterions becomes large enough to invert the cumulative current $j_{\mathrm{cm}}(z)$ from positive (blue) to negative (red), generating a net anionic current $I=j_{\mathrm{cm}}\left(z=d-a_{\mathrm{ns}}\right)<0$ through the pore. This effect is illustrated in the inset of Figure 2.

Thus, the streaming current reversal is the collective effect of $\mathrm{CI}$ and the reduced interfacial liquid mobility. Figure 5a displays the additional role of repulsive image charge forces in terms of the streaming conductance versus the $\mathrm{Spm}^{4+}$ concentration. The comparison of the curves at different membrane permittivities shows that the decrease of the permittivity at a fixed $\mathrm{Spm}^{4+}$
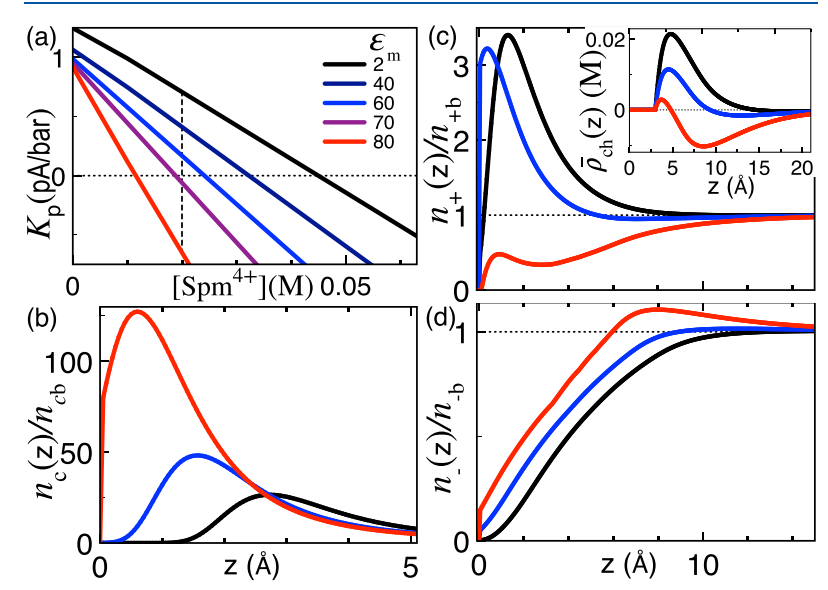

Figure 5. (a) Streaming conductance (eq 40) vs the bulk $\mathrm{Spm}^{4+}$ concentration. (b) Local Spm ${ }^{4+}$ and $(c, d)$ monovalent ion densities (main plots) and the normalized charge flux $\bar{\rho}_{\mathrm{ch}}(z)=\rho_{\mathrm{ch}}(z) u_{\mathrm{c}}(z) / u_{\mathrm{c}}(d /$ 2) (inset) at the bulk Spm ${ }^{4+}$ concentration $n_{\mathrm{cb}}=0.02 \mathrm{M}$ (dashed line in (a)). The bulk salt density is $n_{+\mathrm{b}}=1.0 \mathrm{M}$ in all plots. The membrane permittivity $\varepsilon_{\mathrm{m}}$ for each color is reported in the legend of (a). The remaining model parameters are the same as in Figure 2. 
concentration suppresses the current reversal. As a result, lower substrate permittivities lead to the occurrence of the current reversal at larger $\mathrm{Spm}^{4+}$ concentrations, i.e., $\varepsilon_{\mathrm{m}} \downarrow n_{\mathrm{cb}} \uparrow$.

Figure $5 \mathrm{~b}-\mathrm{d}$ shows that the repulsive polarization forces amplified by the reduction of the substrate permittivity bring two major effects canceling the current inversion. First, the image charge interactions reject the $\mathrm{Spm}^{4+}$ ions from the slit $\left(\varepsilon_{\mathrm{m}}\right.$ $\left.\downarrow n_{\mathrm{c}}(z) \downarrow\right)$, which reduces the $\mathrm{Cl}^{-}$density and raises the $\mathrm{K}^{+}$ density $\left(n_{-}(z) \downarrow n_{+}(z) \uparrow\right)$. Then, the repelled $\mathrm{Spm}^{4+}$ ions move from the no-slip layer toward the hydrodynamically mobile region. These two effects enhance the electrohydrodynamic weight of the $\mathrm{Spm}^{4+}$ and $\mathrm{K}^{+}$cations with respect to the $\mathrm{Cl}^{-}$ anions, which turns the charge flux from negative back to positive (see the inset) and suppresses the current reversal. Next, we consider the opposite case of attractive polarization forces emerging in the giant permittivity regime of surface-coated membrane nanoslits.

III.B. Polarization-Induced Streaming Currents and Ion Separation through Surface-Coated Pores. The surface coating of low permittivity membranes by CNTs is known to enhance the dielectric permittivity of the substrate into the giant permittivity regime $\varepsilon_{\mathrm{m}} \gg \varepsilon_{\mathrm{w}}$ characterized by attractive polarization forces. ${ }^{39}$ Here, we reveal a new mechanism of streaming current generation triggered by the action of these forces on the multivalent ions of the electrolyte mixture.

Figure 6a displays the permittivity dependence of the streaming conductance in neutral slits $\left(\sigma_{\mathrm{m}}=0\right)$ containing the electrolyte mixtures $\mathrm{KCl}+\mathrm{PO}_{4}^{3-}$ (top plot) and $\mathrm{KCl}+\mathrm{Spd}^{3+}$
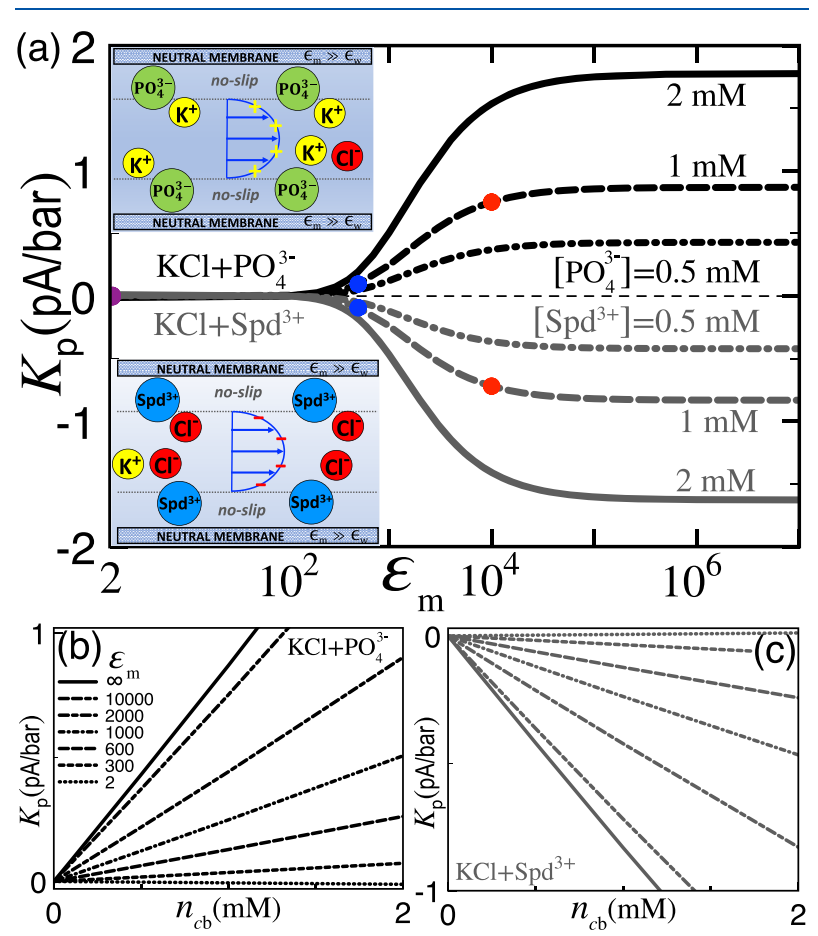

Figure 6. (a) Polarization-induced streaming current against the permittivity of the neutral membrane and $(b, c)$ the multivalent ion concentration in the mixed solutions $\mathrm{KCl}+\mathrm{PO}_{4}^{3-}$ (black curves) and $\mathrm{KCl}+\mathrm{Spd}^{3+}$ (gray curves). The insets in (a) depict the charge configuration at the origin of the current. The steric ion size and salt concentration are $a_{\mathrm{st}}=1.5 \AA$ and $n_{+\mathrm{b}}=0.1 \mathrm{M}$. The other parameters are the same as in Figure 2. (bottom plot) with the trivalent phosphate $\left(\mathrm{PO}_{4}^{3-}\right)$ anions and spermidine $\left(\mathrm{Spd}^{3+}\right)$ cations. In a neutral slit confining a pure $\mathrm{KCl}$ solution, due to the symmetric charge partition, the local charge density (eq 36) and net current (eq 37) would vanish. However, Figure 6a shows that, in the presence of trivalent ions, the rise of the membrane permittivity from the insulator $\varepsilon_{\mathrm{m}} \ll \varepsilon_{\mathrm{w}}$ to the giant permittivity regime $\varepsilon_{\mathrm{m}} \gg \varepsilon_{\mathrm{w}}$ amplifies the streaming conductance from a vanishingly small magnitude to a substantially positive value for $\mathrm{PO}_{4}^{3-}$ anions and a negative value for $\mathrm{Spd}^{3+}$ cations. Moreover, in the metallic regime, $\varepsilon_{\mathrm{m}} \gtrsim$ $10^{5}$, the current amplitude saturates at a limiting value roughly proportional to the multivalent ion concentration. This point is explicitly illustrated in Figure 6b,c: for $\varepsilon_{\mathrm{m}}>\varepsilon_{\mathrm{w}}$ where added $\mathrm{PO}_{4}^{3-}$ anions ( $\mathrm{Spd}^{3+}$ cations) into the $\mathrm{KCl}$ solution activate a positive (negative) conductance whose magnitude rises with the multivalent ion density $\left(K_{\mathrm{p}} \propto n_{\mathrm{cb}}\right)$ and the membrane permittivity $\left(\varepsilon_{\mathrm{m}} \uparrow\left|K_{\mathrm{p}}\right| \uparrow\right)$.

Thus, in the giant permittivity regime of the surface-coated pores, the attractive polarization forces coupled to the multivalent ions can solely set a counterion current whose sign and strength can be tuned by the type and amount of the added multivalent charges. This effect is the key prediction of our work. Figure 7 illustrates the mechanism driving this current in terms
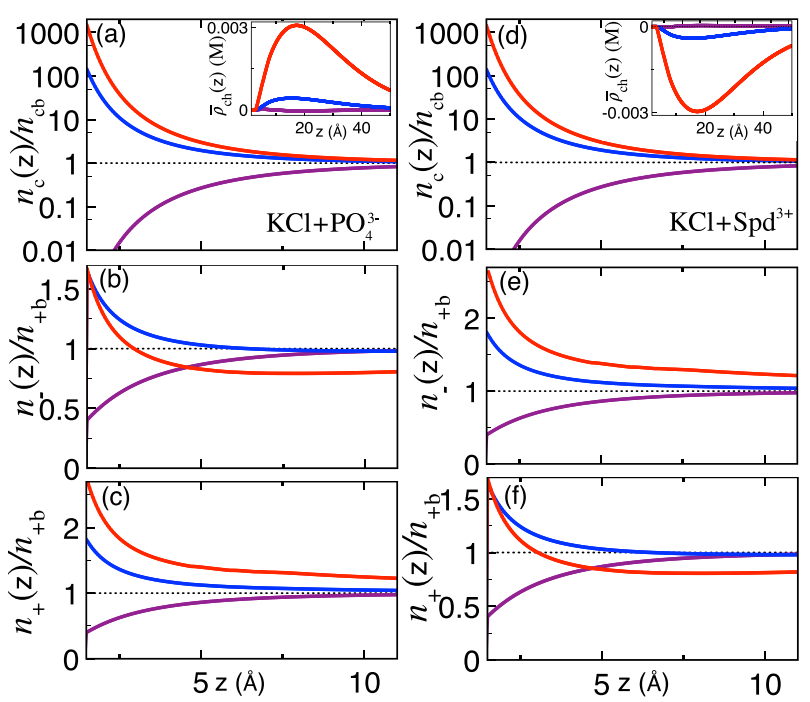

Figure 7. (Color online) (a) Multivalent counterion density (eq 26) (main plot) together with the normalized charge flux $\bar{\rho}_{\mathrm{ch}}(z)=\rho_{\mathrm{ch}}(z)$ $u_{\mathrm{c}}(z) / u_{\mathrm{c}}(d / 2)$ (inset) and (b, c) the monovalent ion densities (eq 25) for the $\mathrm{KCl}+\mathrm{PO}_{4}^{3-}$ mixture confined to a neutral slit pore. The permittivity values corresponding to the dots of the same color in Figure 6 are $\varepsilon_{\mathrm{m}}=2$ (purple), 500 (blue), and $10^{4}$ (red). (d-f) Equivalent plots for the $\mathrm{KCl}+\mathrm{Spd}^{3+}$ liquid.

of the mono- and multivalent ion densities, and the normalized charge flux for the $\mathrm{KCl}+\mathrm{PO}_{4}^{3-}$ solution (left) and the $\mathrm{KCl}+$ $\mathrm{Spd}^{3+}$ mixture (right). The permittivity value for each curve corresponds to the dots of the same color in Figure 6a.

The plots indicate that the increment of the membrane permittivity from the repulsive (purple) to the attractive image charge regime $\varepsilon_{\mathrm{m}}=500$ (blue) switches the configuration of all ionic species from the exclusion $\left(n_{ \pm, c}(z)<n_{ \pm, \mathrm{cb}}\right)$ to the excess state $\left(n_{ \pm, \mathrm{c}}(z)>n_{ \pm, \mathrm{cb}}\right)$. However, according to Figure 6a, the substantial amplification of the pore conductance by polarization forces occurs in the subsequent permittivity regime $\varepsilon_{\mathrm{m}} \gtrsim$ 500. Indeed, Figure 7 shows that, due to the quadratic 
dependence of the attractive image charge interactions on the ion valency (see eqs 22 and 23), the multivalent ions experience a significantly stronger interfacial adsorption than the monovalent salt ions. In the permittivity regime $\varepsilon_{\mathrm{m}} \gtrsim 500$ where this asymmetry becomes pronounced, the salt-multivalent ion interactions take over the salt-image charge interactions. Consequently, the $\mathrm{PO}^{3-}\left(\mathrm{Spd}^{3+}\right)$ adsorption attracts further $\mathrm{K}^{+}\left(\mathrm{Cl}^{-}\right)$ions but excludes the $\mathrm{Cl}^{-}\left(\mathrm{K}^{+}\right)$ions from the slit (red curves). The insets in Figure 7a,d show that this results in a charge flux opposite to the sign of the added multivalent charges, explaining the counterion current displayed in Figure 6. The corresponding charge separation mechanism is schematically depicted in the insets of Figure 6.

Considering the difficulty in modifying externally the sign and density of the nanopore surface charges, the polarizationinduced streaming current presents itself as a highly relevant prediction for nanofluidic ion separation purposes. Figure $8 \mathrm{a}, \mathrm{b}$

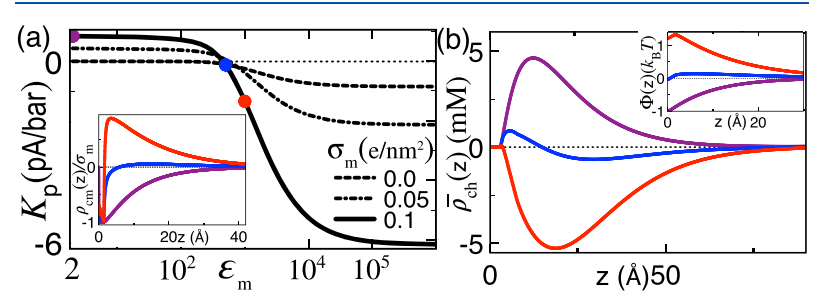

Figure 8. (a) Permittivity dependence of the streaming conductance at different anionic surface charges $\sigma_{\mathrm{m}}$ (main plot) and the cumulative charge density (eq 42) (inset) for the $\mathrm{KCl}+\mathrm{Spd}^{3+}$ liquid. (b) Normalized charge flux (main plot) and the average potential (eq 34) (inset). The membrane permittivities of the curves corresponding to the dots of the same color in (a) are $\varepsilon_{\mathrm{m}}=2$ (purple), 500 (blue), and $10^{3}$ (red). The ion concentrations are $n_{+\mathrm{b}}=0.1 \mathrm{M}$ and $n_{\mathrm{cb}}=1.0 \mathrm{mM}$. The other parameters are the same as in Figure 2.

illustrates the extension of this mechanism to membranes with a finite anionic surface charge. One sees that, in negatively charged slits, the increase of the membrane permittivity from the insulator to the giant permittivity regime leads to the pore CI (see the insets) and the reversal of the charge flux and conductance from a finite positive to a negative value (see the main plots). It is noteworthy that the activation of surface CI by the exclusive effect of the attractive polarization forces has been previously demonstrated in the MC simulations of multivalent electrolytes in contact with planar conductors. ${ }^{49}$ This partial overlap of the results is a relevant numerical support of the polarization-induced current generation mechanism revealed herein.

III.C. Ion Separation by Attractive Polarization Forces in Voltage-Driven Charge Transport. The voltage-induced charge transport driven by the individual ion conductivities radically differs from the pressure-driven convective transport process studied in Sec. III.B. We probe here the effect of the attractive coupling between the polarization forces and the multivalent ions on the ionic composition of voltage-driven currents. We consider a neutral slit of small thickness $d=5 \mathrm{~nm}$ where deviations from the bulk transport behavior can be easily observed. In order to focus on the ion separation regime, the bulk multivalent ion concentration will be set to a sufficiently large value for the monovalent coions to be quasi-totally depleted from the slit.

Figure 9a displays the dielectric permittivity dependence of the conductance (eq 41) rescaled by its bulk value

$$
K_{\mathrm{Vb}}=\frac{w \mathrm{e}}{L}\left(d-2 a_{\mathrm{ns}}\right) \sum_{i= \pm, \mathrm{c}} \mu_{i}\left|q_{i}\right| n_{i \mathrm{~b}}
$$

for the $\mathrm{KCl}$ and $\mathrm{KCl}+\mathrm{Spm}^{4+}$ solutions. As the permittivity rises from the insulator to the metallic limit, the multivalent ion effects come into play in the giant permittivity regime $\varepsilon_{\mathrm{m}} \gtrsim 10^{3}$ where the $\mathrm{KCl}+\mathrm{Spm}^{4+}$ conductance (solid blue curve) experiences a significantly stronger enhancement than the $\mathrm{KCl}$ conductance (black). In Figure 9a, we reported as well the reduced conductance $\left(K_{\mathrm{V}}-K_{\mathrm{V}, \mathrm{c}}\right) / K_{\mathrm{Vb}}$ excluding the $\mathrm{Spm}^{4+}$
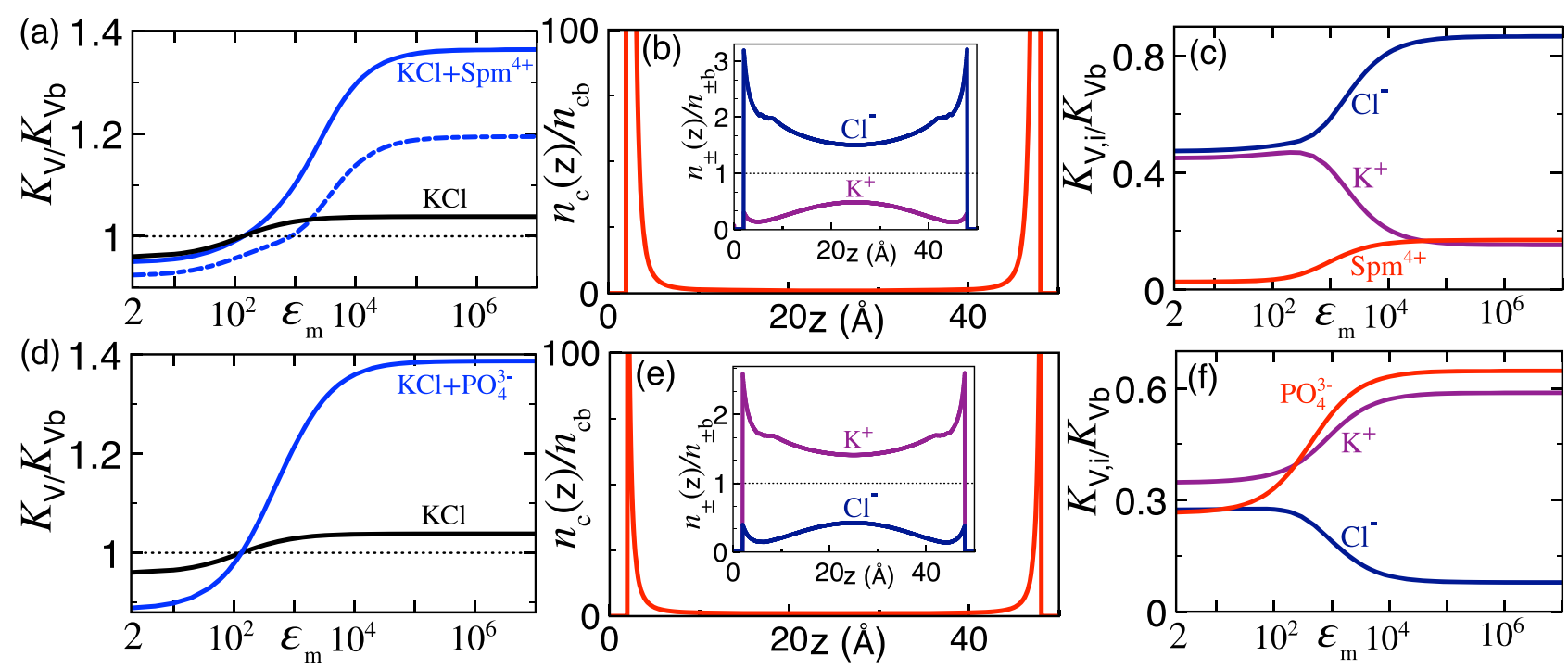

Figure 9. (a) Dielectric permittivity dependence of the voltage-driven conductance (eq 41) for the $\mathrm{KCl}$ solution (black curve) and the electrolyte mixture $\mathrm{KCl}+\mathrm{Spm}^{4+}$ (solid blue curve). (b) $\mathrm{Spm}^{4+}$ (main plot) and monovalent salt densities (inset) at the membrane permittivity $\varepsilon_{\mathrm{m}}=10^{7}$. (c) Conductance components (eq 46) of separate species. The dashed blue curve in (a) displays the $\mathrm{KCl}+\mathrm{Spm}^{4+}$ conductance without the Spm ${ }^{4+}$ component, i.e., $\left(K_{\mathrm{V}}-K_{\mathrm{V}, \mathrm{c}}\right) / K_{\mathrm{Vb}}$. The neutral pore size is $d=5 \mathrm{~nm}$, the steric ion size $a_{\mathrm{st}}=2.0 \AA$, and the ion concentrations are $n_{\mathrm{bb}}=0.1 \mathrm{M}$ and $n_{\mathrm{cb}}=$ $0.5 \mathrm{mM}$. (d-f) Equivalent plots for the $\mathrm{KCl}+\mathrm{PO}_{4}^{3-}$ mixture with $n_{\mathrm{cb}}=10.0 \mathrm{mM}$. The remaining parameters are the same as in Figure 2. 
contribution (dashed curve) with the conductance components of the separate ionic species $i=\{ \pm, c\}$ defined as

$$
K_{V, i}=\frac{w e}{L} \mu_{i}\left|q_{i}\right| \int_{a_{n s}}^{d-a_{n s}} \mathrm{~d} z n_{i}(z)
$$

One sees that, upon the increment of the membrane permittivity from $\varepsilon_{\mathrm{m}} \approx 2$ to $\varepsilon_{\mathrm{m}} \gg \varepsilon_{\mathrm{w}}$, the reduced and total conductances are amplified by comparable amounts. This suggests that the principle role played by the $\mathrm{Spm}^{4+}$ cations on the current enhancement is electrostatic.

The primarily electrostatic effect of the $\mathrm{Spm}^{4+}$ ions on the current amplification is corroborated in Figure 9b,c. According to Figure $9 \mathrm{~b}$, for $\varepsilon_{\mathrm{m}} \gg \varepsilon_{\mathrm{w}}$, the $\mathrm{Spm}^{4+}$ cations strongly adsorbed by the pore walls exclude the $\mathrm{K}^{+}$cations and attract the $\mathrm{Cl}^{-}$ anions. Figure $9 \mathrm{c}$ shows that, beyond the permittivity value $\varepsilon_{\mathrm{m}} \approx$ $10^{3}$, this leads to the suppression of the $\mathrm{K}^{+}$conductivity $\left(\varepsilon_{\mathrm{m}} \uparrow\right.$ $\left.K_{\mathrm{V},+} \downarrow\right)$ and the enhancement of the $\mathrm{Cl}^{-}$conductivity $\left(\varepsilon_{\mathrm{m}} \uparrow\right.$ $\left.K_{\mathrm{V},-} \uparrow\right)$ responsible for the amplification of the $\mathrm{KCl}+\mathrm{Spm}^{4+}$ conductance observed in Figure 9a. Although attractive polarization forces equally amplify the $\mathrm{Spm}^{4+}$ conductance (red curve in Figure 9c), due to the low bulk Spm ${ }^{4+}$ density, the former is largely dominated by the enhanced $\mathrm{Cl}^{-}$component. Thus, in the giant permittivity regime of surface-coated pores, added $\mathrm{Spm}^{4+}$ ions induce an electrostatic charge discrimination and generate a $\mathrm{Cl}^{-}$-rich current without making themselves a substantial conductive contribution to charge transport.

Figure $9 \mathrm{~d}$ shows that the addition of the trivalent $\mathrm{PO}_{4}^{3-}$ anions to the $\mathrm{KCl}$ solution leads to a quantitatively comparable enhancement of the total conductance, which is partly driven by the direct $\mathrm{PO}_{4}^{3-}-\mathrm{K}^{+}$interactions. Namely, in the regime $\varepsilon_{\mathrm{m}} \gg \varepsilon_{\mathrm{w}}$, the adsorbed $\mathrm{PO}_{4}^{3-}$ ions setting an anionic surface charge exclude the $\mathrm{Cl}^{-}$anions and attract the $\mathrm{K}^{+}$cations (see Figure 9e). At $\varepsilon_{\mathrm{m}} \gtrsim 10^{3}$, this reduces the $\mathrm{Cl}^{-}$conductivity and enhances the $\mathrm{K}^{+}$conductivity, i.e., $\varepsilon_{\mathrm{m}} \uparrow K_{\mathrm{V},-} \downarrow K_{\mathrm{V},+} \uparrow$ (see Figure 9f). However, in contrast with the previous case of $\mathrm{Spm}^{4+}$ charges, the $\mathrm{PO}_{4}^{3-}$ ions of lower valency and larger bulk concentrations also bring a sizable conductive contribution to the increment of the total current; one notes that, in the giant permittivity regime, the $\mathrm{PO}_{4}^{3-}$ conductance becomes even higher than the $\mathrm{K}^{+}$conductance. Hence, the separate electrostatic and conductive contributions from the $\mathrm{PO}_{4}^{3-}$ ions lead to a voltagedriven current equally rich in the $\mathrm{K}^{+}$and $\mathrm{PO}_{4}^{3-}$ species.

\section{CONCLUSIONS}

The comprehension of charged systems characterized by the omnipresence of composite electrolytes requires the formulation of electrostatic theories able to take into account mixed interaction strengths. Via the self-consistent incorporation of dilute multivalent ions into the PB equation, we developed a theoretical formalism accounting for the electrostatic and $\mathrm{HC}$ interactions of WC monovalent salt and SC multivalent ions. By combining the corresponding SCPB formalism with the Stokes equation, we investigated the impact of SC electrostatics on nanofluidic charge transport through nanoslits fabricated in lowpermittivity silica and surface-coated dielectric membranes.

The SCPB theory was applied to characterize the emergence of negative streaming currents in strongly anionic silica nanochannels. ${ }^{9}$ This exotic transport behavior is driven by the collective effect of the multivalent counterions activating the membrane $\mathrm{CI}$ and bringing $\mathrm{Cl}^{-}$ions into the slit and the no-slip zone reducing the contribution from these interfacial counterions to the net current. As a result of this cooperative mechanism, the like-charge current formation requires the inversion of the average potential within the no-slip region.

We have also probed SC effects on the pressure-driven transport properties of surface-coated nanopores located in the giant permittivity regime $\varepsilon_{\mathrm{m}} \gg \varepsilon_{\mathrm{w}}$ associated with attractive polarization forces. ${ }^{39}$ Under the effect of these forces, added multivalent ions result in a charge separation and generate a counterion current through interfacially neutral slits. This key prediction can be readily verified by transport experiments and the underlying electrohydrodynamic mechanism can be beneficial to ion separation and water purification technologies.

In addition, we found that, if the nanoslit carries anionic surface charges, the increment of the membrane permittivity from the insulator to the giant permittivity regime can solely trigger the pore $\mathrm{CI}$ and reverse the streaming current from positive to negative. Hence, the polarization-driven current generation mechanism is supported by MC simulations where the activation of CI by the exclusive effect of the attractive image-charge interactions has been previously observed. ${ }^{49}$

In the case of voltage-driven transport through neutral dielectric slits, charge separation by quadrivalent $\mathrm{Spm}^{4+}$ molecules generates a single-species current rich in $\mathrm{Cl}^{-}$. However, if the charge separation is achieved by the addition of trivalent $\mathrm{PO}_{4}^{3-}$ ions, their higher bulk concentration resulting from their lower valency leads to a voltage-driven current equally rich in the $\mathrm{PO}_{4}^{3-}$ and $\mathrm{K}^{+}$species. Thus, in the charge-separated current, the weight of the multivalent ions varies inversely with their valency.

The consequences of the polarization-induced charge separation on the field-driven polymer translocation ${ }^{21}$ will be explored in an upcoming work. We also note that the present formalism was based on the assumption of a uniform no-slip length. In future work, this approximation can be relaxed via the consideration of the explicit ion or solvent effects on the interfacial liquid viscosity. ${ }^{15}$ Finally, in the Supporting Information, the SCPB formalism is shown to provide a selfconsistent route to the dressed-ion theory. ${ }^{34,35}$ In light of this exact mapping, the comparative confrontation of these formalisms with intensive MC simulations will help us to identify the validity regime of the underlying approximations.

\section{ASSOCIATED CONTENT}

\section{Supporting Information}

The Supporting Information is available free of charge at https://pubs.acs.org/doi/10.1021/acs.jpcb.0c09638.

Green's function in slit pores, perturbative solution of the SCPB equation, identification of the membrane charge reversal conditions from the dressed-in approach, and Figure S1: comparison of the cumulative charge density obtained from the dressed-ion theory ${ }^{35}$ and the SCPB formalism with MC simulation data (PDF)

\section{AUTHOR INFORMATION}

\section{Corresponding Author}

Sahin Buyukdagli - Department of Physics, Bilkent University, Ankara 06800, Turkey; @ orcid.org/0000-0002-2133470X; Email: buyukdagli@fen.bilkent.edu.tr

Complete contact information is available at:

https://pubs.acs.org/10.1021/acs.jpcb.0c09638

\section{Notes}

The author declares no competing financial interest. 


\section{ACKNOWLEDGMENTS}

The author received no financial support for this work.

\section{REFERENCES}

(1) Holm, C.; Kékicheff, P.; Podgornik, R. Electrostatic Effects in Soft Matter and Biophysics; Springer Science \& Business Media: Dordrecht, 2001.

(2) Schoch, R. B.; Han, J.; Renaud, P. Transport Phenomena in Nanofluidics. Rev. Mod. Phys. 2008, 80, 839-883.

(3) Barrat, J.-L.; Bocquet, L. Large Slip Effect at a Nonwetting FluidSolid Interface. Phys. Rev. Lett. 1999, 82, 4671-4674.

(4) Sendner, C.; Horinek, D.; Bocquet, L.; Netz, R. R. Interfacial Water at Hydrophobic and Hydrophilic Surfaces: Slip, Viscosity, and Diffusion. Langmuir 2009, 25, 10768-10781.

(5) Bocquet, L.; Charlaix, E. Nanofluidics, from Bulk to Interfaces. Chem. Soc. Rev. 2010, 39, 1073-1095.

(6) Bocquet, L. Nanofluidics Coming of Age. Nat. Mater. 2020, 19, 254-256.

(7) Yaroshchuk, A. E. Non-steric Mechanism of Nanofiltration: Superposition of Donnan and Dielectric Exclusion. Sep. Purif. Technol. 2001, 22-23, 143-158.

(8) Levin, Y. Electrostatics of Ions Inside the Nanopores and TransMembrane Channels. Europhys. Lett. 2006, 76, 163-169.

(9) van der Heyden, F. H. J.; Stein, D.; Dekker, C. Streaming Currents in a Single Nanofluidic Channel. Phys. Rev. Lett. 2005, 95, 116104.

(10) Daiguji, H.; Yang, P.; Szeri, A. J.; Majumdar, A. Electrochemomechanical Energy Conversion in Nanofluidic Channels. Nano Lett. 2004, 4, 2315-2321.

(11) van der Heyden, F. H. J.; Bonthuis, D. J.; Stein, D.; Meyer, C.; Dekker, C. Electrokinetic energy conversion efficiency in nanofluidic channels. Nano Lett. 2006, 6, 2232-2237.

(12) van der Heyden, F. H. J.; Bonthuis, D. J.; Stein, D.; Meyer, C.; Dekker, C. Power Generation by Pressure-Driven Transport of Ions in Nanofluidic Channels. Nano Lett. 2007, 7, 1022-1025.

(13) Gillespie, D. High Energy Conversion Efficiency in Nanofluidic Channels. Nano Lett. 2012, 12, 1410-1416.

(14) Gillespie, D.; Pennathur, S. Separation of Ions in Nanofluidic Channels with Combined Pressure-Driven and Electro-Osmotic Flow. Anal. Chem. 2013, 85, 2991-2998.

(15) Rezaei, M.; Azimian, A. R.; Pishevar, A. R.; Bonthuis, D. J. Viscous interfacial layer formation causes electroosmotic mobility reversal in monovalent electrolytes. Phys. Chem. Chem. Phys. 2018, 20, $22517-22524$

(16) Uematsu, Y.; Araki, T. Electro-osmotic Flow of Semidilute Polyelectrolyte Solutions. J. Chem. Phys. 2013, 139, No. 094901.

(17) Moreira, A. G.; Netz, R. R. Strong-coupling Theory for Counterion Distributions. Europhys. Lett. 2000, 52, 705-711.

(18) van der Heyden, F. H. J.; Stein, D.; Besteman, K.; Lemay, S. G.; Dekker, C. Charge Inversion at High Ionic Strength Studied by Streaming Currents. Phys. Rev. Lett. 2006, 96, 224502.

(19) Qiu, S.; Wang, Y.; Cao, B.; Guo, Z.; Chen, Y.; Yang, G. The Suppression and Promotion of DNA Charge Inversion by Mixing Counterions. Soft Matter 2015, 11, 4099-4105.

(20) Sugimoto, T.; Nishiya, M.; Kobayashi, M. Charge Reversal of Sulfate Latex Particles in the Presence of Lanthanum Ion. Colloids Surf., A 2019, 572, 18-26.

(21) Buyukdagli, S. Facilitated Polymer Capture by Charge Inverted Electroosmotic Flow in Voltage-driven Polymer Translocation. Soft Matter 2018, 14, 3541-3549.

(22) Gillespie, D.; Khair, A. S.; Bardhan, J. P.; Pennathur, S. Efficiently Accounting for Ion Correlations in Electrokinetic Nanofluidic Devices Using Density Functional Theory. J. Colloid Interface Sci. 2011, 359, $520-529$.

(23) Hoffmann, J.; Gillespie, D. Ion Correlations in Nanofluidic Channels: Effects of Ion Size, Valence, and Concentration on Voltageand Pressure-driven Currents. Langmuir 2013, 29, 1303-1317.
(24) Gulbrand, L.; Jönsson, B.; Wennerström, H.; Linse, B. Electrical Double Layer Forces. A Monte Carlo Study. J. Chem. Phys. 1984, 80, 2221-2228.

(25) Besteman, K.; Zevenbergen, M. A. G.; Lemay, S. G. Charge Inversion by Multivalent Ions: Dependence on Dielectric Constant and Surface-charge Density. Phys. Rev. E 2005, 72, No. 061501.

(26) Trulsson, M.; Jönsson, B.; Åkesson, T.; Forsman, J.; Labbez, C. Repulsion Between Oppositely Charged Surfaces in Multivalent Electrolytes. Phys. Rev. Lett. 2006, 97, No. 068302.

(27) Podgornik, R.; Žekš, B. Inhomogeneous Coulomb Fluid. A Functional Integral Approach. J. Chem. Soc., Faraday Trans. 2 1988, 84, 611-631.

(28) Attard, P.; Mitchell, D. J.; Ninham, B. W. Beyond PoissonBoltzmann: Images and Correlations in the Electric Double Layer II. Symmetric Electrolyte. J. Chem. Phys. 1988, 89, 4358.

(29) Netz, R. R.; Orland, H. Beyond Poisson-Boltzmann: Fluctuation Effects and Correlation Functions. Eur. Phys. J. E: Soft Matter Biol. Phys. 2000, 1, 203-214.

(30) Lau, A. W. C. Fluctuation and Correlation Effects in a Charged Surface Immersed in an Electrolyte Solution. Phys. Rev. E 2008, 77, No. 011502

(31) Buyukdagli, S.; Achim, C. V.; Ala-Nissila, T. Electrostatic Correlations in Inhomogeneous Charged Fluids Beyond Loop Expansion. J. Chem. Phys. 2012, 137, 104902.

(32) Buyukdagli, S.; Ala-Nissila, T. Electrostatic Correlations on the Ionic Selectivity of Cylindrical Membrane Nanopores. J. Chem. Phys. 2014, 140, No. 064701.

(33) Buyukdagli, S.; Blossey, R.; Ala-Nissila, T. Ionic Current Inversion in Pressure-driven Polymer Translocation through Nanopores. Phys. Rev. Lett. 2015, 114, No. 088303.

(34) Kanduĉ, M.; Naji, A.; Forsman, J.; Podgornik, R. Dressed Counterions: Strong Electrostatic Coupling in the Presence of Salt. J. Chem. Phys. 2010, 132, 124701.

(35) Kanduč, M.; Naji, A.; Forsman, J.; Podgornik, R. Dressed Counterions: Poly- and Monovalent Ions at Charged Dielectric Interfaces. Phys. Rev. E 2011, 84, No. 011502.

(36) Buyukdagli, S.; Podgornik, R. Like-charge Polymer-membrane Complexation Mediated by Multivalent Cations: One-loop-dressed Strong Coupling Theory. J. Chem. Phys. 2019, 151, No. 094902.

(37) Buyukdagli, S. Schwinger-Dyson Equations for Composite Electrolytes Governed by Mixed Electrostatic Coupling Strengths. J. Chem. Phys. 2020, 152, No. 014902.

(38) Storey, B. D.; Bazant, M. Z. Effects of Electrostatic Correlations on Electrokinetic Phenomena. Phys. Rev. E 2012, 86, No. 056303.

(39) Yuan, J.-K.; Yao, S.-H.; Dang, Z.-M.; Sylvestre, A.; Genestoux, M.; Bai, J. Giant Dielectric Permittivity Nanocomposites: Realizing True Potential of Pristine Carbon Nanotubes in Polyvinylidene Fluoride Matrix through an Enhanced Interfacial Interaction. J. Phys. Chem. C 2011, 115, 5515-5521.

(40) Moreira, A. G.; Netz, R. R. Virial Expansion for Charged Colloids and Electrolytes. Eur. Phys. J. D 2002, 21, 83-96.

(41) Hansen, J.-P.; McDonald, I. R. Theory of Simple Liquids: with Applications to Soft Matter; Academic Press: 2013.

(42) The potential $\phi-_{\mathrm{s}}(\mathrm{r})$ solving the SCPB Eq. (30) is screened exclusively by the monovalent salt; therein, the multivalent counterions interfere indirectly via their scattering with the monovalent ions.

(43) The monovalent ion mobilities are set to the experimental values of $\mu_{+}=7.616 \times 10^{-8} \mathrm{~m}^{2} / \mathrm{V}^{-1} \mathrm{~s}^{-1}$ for $\mathrm{K}^{+}$and $\mu=7.909 \times 10^{-8} \mathrm{~m}^{2} \mathrm{~V}^{-1} \mathrm{~s}^{-1}$ for $\mathrm{Cl}^{-44}$ To our knowledge, the drift transport mobilities of the phosphate $\mathrm{PO}_{4}^{3-}$, spermidine $\mathrm{Spm}^{3+}$, and spermine $\mathrm{Spm}^{4+}$ charges are not available in the literature. Based on the Einstein relation $\mu_{i}=\left|\mathrm{q}_{i}\right| D /\left(k_{\mathrm{B}} T\right)$, the transport coefficient of these charges were estimated as $\mu_{3+}=3 \mu_{+}$and $\mu_{4+}=4 \mu_{+}$

(44) Lide, D. R. CRC Handbook of Chemistry and Physics; 93th edition, CRC Press: 2004

(45) Butt, H.-J.; Kappl, M. Surface and Interfacial Forces; Wiley-VCH: 2010.

(46) Sánchez-Arellano, E.; Olivares, W.; Lozada-Cassou, M.; JiménezÁngeles, F. Electrokinetic Properties of Monovalent Electrolytes 
Confined in Charged Nanopores: Effect of Geometry and Ionic Shortrange Correlations. J. Colloid Interface Sci. 2009, 330, 474-482.

(47) Buyukdagli, S.; Ala-Nissila, T. Controlling Polymer Translocation and ion Transport via Charge Correlations. Langmuir 2014, 30, 12907-12915.

(48) In the Supporting Information, a self-consistent rederivation of the dressed-ion theory ${ }^{35}$ from the SCPB Eq. (30) is carried out. Then, within the dressed-ion approach, it is shown that in contrast with the current reversal phenomenon, $\mathrm{CI}$ occurs in the presence of any amount of added multivalent counterions at large enough separation distances from the membrane. This result is an additional argument indicating the absence of one-to-one mapping between $\mathrm{CI}$ and current reversal.

(49) Wang, Z.-Y. Image-induced overcharging in the weakly charged surfaces. J. Stat. Mech.: Theory Exp. 2016, No. 043205. 